\title{
Shell Filling and Spin Effects in a Few Electron Quantum Dot
}

\author{
S. Tarucha, D. G. Austing, and T. Honda \\ NTT Basic Research Laboratories, 3-1, Morinosoto Wakamiya, Atsugi-shi, Kanagawa 243-01, Japan \\ R. J. van der Hage and L.P. Kouwenhoven \\ Department of Applied Physics, Delft University of Technology, PO Box 5046, 2600 GA Delft, The Netherlands \\ (Received 20 May 1996)
}

\begin{abstract}
We study atomiclike properties of artificial atoms by measuring Coulomb oscillations in vertical quantum dots containing a tunable number of electrons starting from zero. At zero magnetic field the energy needed to add electrons to a dot reveals a shell structure for a two-dimensional harmonic potential. As a function of magnetic field the current peaks shift in pairs, due to the filling of electrons into spin-degenerate single-particle states. When the magnetic field is sufficiently small, however, the pairing is modified, as predicted by Hund's rule, to favor the filling of parallel spins. [S00319007(96)01418-4]
\end{abstract}

PACS numbers: 73.20.Dx, 72.20.My, 73.40.Gk

The "addition energy" needed to place an extra electron in a semiconductor quantum dot is analogous to the electron affinity for a real atom [1]. For a fixed number of electrons, small energy excitations can take these electrons to a higher single-particle state. However, due to Coulomb interactions between the electrons, the addition energy is greater than the energy associated with these excitations. Both the addition energy spectrum and the excitation energy spectrum are discrete when the Fermi wavelength and the dot size are comparable. Until now a direct mapping of the observed addition energy, and the single-particle excitation energy, to a calculated spectrum has been hampered, probably due to sample specific inhomogeneities [2].

The three-dimensional spherically symmetric potential around atoms gives rise to the shell structure $1 s, 2 s, 2 p$, $3 s, 3 p, \ldots$. The ionization energy has a large maximum for atomic numbers $2,10,18, \ldots$ Up to atomic number 23 these shells are filled sequentially, and Hund's rule determines whether a spin-down or a spin-up electron is added [3]. Vertical quantum dots have the shape of a disk with a diameter roughly 10 times the thickness $[2,4]$. The lateral potential has a cylindrical symmetry with a rather soft boundary profile, which can be approximated by a harmonic potential. The symmetry of this twodimensional (2D) harmonic potential leads to a complete filling of shells for $2,6,12, \ldots$ electrons. The numbers in this sequence can be regarded as "magic numbers" for a 2D harmonic dot. The shell filling in this manner is previously predicted by self-consistent calculations of a circular dot [5]. In this Letter we report the observation of atomiclike properties in the conductance characteristics of a vertical quantum dot. We find an unusually large addition energy when the electron number coincides with a magic number. We can identify the quantum numbers of the single-particle states by studying the magnetic field dependence. At a sufficiently small magnetic field $(B<0.4 \mathrm{~T})$ we see that spin filling obeys Hund's rule. At higher magnetic fields $(B>0.4 \mathrm{~T})$ we observe the consecutive filling of states by spin-up and spin-down electrons, which arises from spin degeneracy.

The gated vertical quantum dot shown schematically in Fig. 1 is made from a double-barrier heterostructure (DBH). The use of well-defined heterostructure tunnel junctions allows us to vary the number of electrons in the dot $N$ one by one from 0 to more than 40 by changing
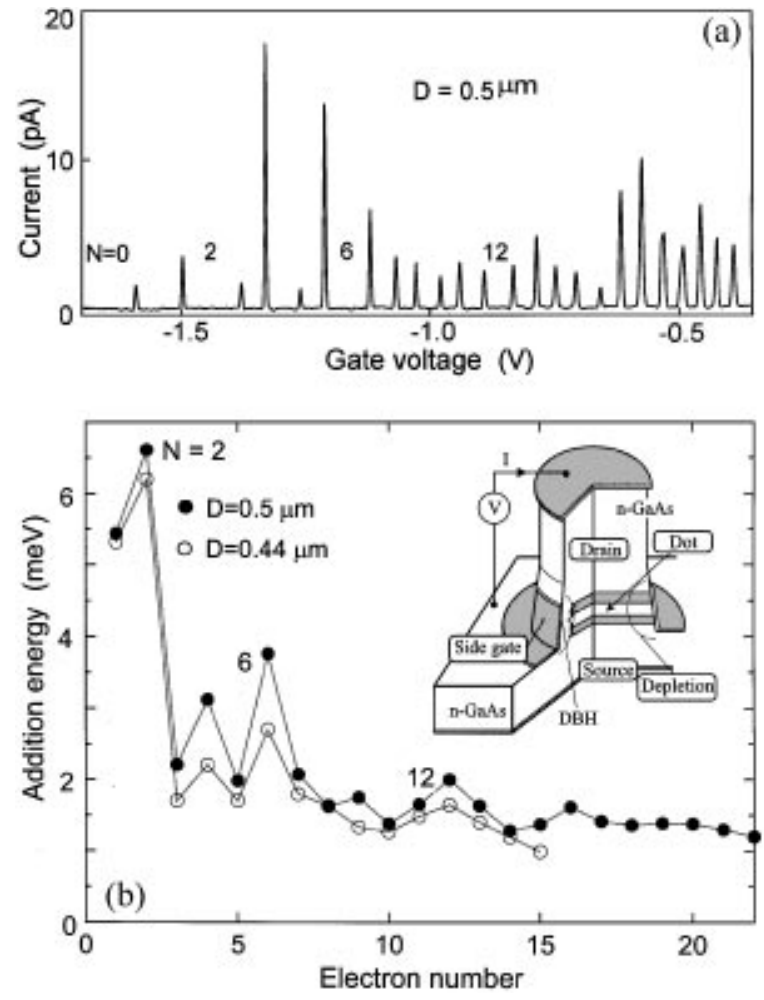

FIG. 1. (a) Coulomb oscillations in the current vs gate votage at $B=0 \mathrm{~T}$ observed for a $D=0.5 \mu \mathrm{m}$ dot. (b) Addition energy vs electron number for two different dots with $D=0.5$ and $0.44 \mu \mathrm{m}$. The inset shows a schematic diagram of the device. The dot is located between the two heterostructure barriers. 
the gate voltage $V_{g}$ [4]. The DBH consists of an undoped 12.0-nm $\mathrm{In}_{0.05} \mathrm{Ga}_{0.95}$ As well and undoped $\mathrm{Al}_{0.22} \mathrm{Ga}_{0.78} \mathrm{As}$ barriers of thickness 9.0 and $7.5 \mathrm{~nm}$ (the thinner one is closest to the substrate). The source and drain contacts are made from $n$-GaAs and are lightly doped close to the $\mathrm{DBH}$. The DBH is processed to form a mesa with top contact geometrical diameter $D$ by using a combined dry and wet etch to a point just below the DBH region. A circular Schottky gate is placed on the side of the mesa close to the DBH [4]. We point out that the inclusion of In in the well reduces the bottom of the conduction band below the Fermi level of the contacts. This allows us to study linear transport through a vertical quantum dot. The current $I$ flowing vertically through the dot is measured in response to a small dc voltage $V$ applied between the contacts. Note that all the results are reproduced in both polarities for $V$ since the device is in the linear transport regime. The samples are cooled in a dilution refrigerator down to $50 \mathrm{mK}$, although the electron temperature is estimated to be about $0.2 \mathrm{~K}$.

Figure 1(a) shows the current at $V=150 \mu \mathrm{V}$ as a function of $V_{g}$ for a dot with $D=0.5 \mu \mathrm{m}$. Clear Coulomb oscillations are observed for $V_{g}>-1.6 \mathrm{~V}$ with each period corresponding to a change of exactly one electron in the dot. From $I-V$ characteristics (not shown) we can unambiguously assign absolute values of $N$, i.e., $N=1$ between the first and second peaks, $N=2$ between the second and third peaks, etc. We find that when $N$ becomes smaller than 20, the oscillation period depends strongly on $N$. The increasing "irregularity" for small $N$ has previously been reported for dots containing a few electrons $[2,4]$, but in marked contrast we find that the irregularity in our dot is, in fact, systematic with respect to $N$.

Figure 1(b) shows the addition energy as a function of $N$ for two different devices. The spacing between the current peaks in Fig. 1(a) reflects the energy to add one more electron to a dot containing $N$ electrons. For example, the energy to add the third electron to an $N=2$ dot can be derived from the spacing between the second and third peaks. For each value of $N$ the factor $\alpha$ to convert gate voltage to addition energy can be determined from the $V_{g}$ dependence of the $I-V$ characteristics [6]. The differential conductance $d I / d V$ plotted in linear grey scale in the $V-V_{g}$ plane reveals a series of diamond shaped regions associated with Coulomb blockade. The boundary of the $N$ th region of the Coulomb blockade is defined by the conditions that the electrochemical potential of the collector and emitter, respectively, align with the electrochemical potential $\mu(N)$ of the dot when $N$ and $N+1$ electrons are trapped in the dot. When the boundary is located at a vanishingly small $V$, the $N$ and $N+1$ peaks occur in the $I-V_{g}$ characteristic. The boundary identifies the linear transport regime, and we can determine the addition energies directly from half-widths of the Coulomb diamonds. The $\alpha$ value determined in this way, for example, in the $D=0.5 \mu \mathrm{m}$ dot, varies from 57 to $42 \mathrm{meV} / \mathrm{V}$ for $N=1$ to 6 , and then gradually decreases to $33 \mathrm{meV} / \mathrm{V}$ as $N$ approaches 20. As $N$ is decreased, the addition energy generally becomes larger due to the increase of the Coulomb interaction as the effective dot size is decreased. We find that the addition energy is unusually large for $N=2,6$, and 12 for these two devices. In eight devices with $D$ between 0.4 and $0.54 \mu \mathrm{m}$ the addition energy is unusually large for $N=2$ and 6. An unusually large addition energy for $N=12$ is observed in three devices. We also observe a relatively large addition energy for $N=4$ in most of the devices. In the remaining part of this Letter we focus on one particular $D=0.5 \mu \mathrm{m}$ device. All the main features, however, have been reproduced in other devices.

The electronic states are expected to be significantly modified by a magnetic field $B$ applied parallel to the tunneling current. We show the $B$-field dependence of the position of the current oscillations in Fig. 2. It is constructed from $I-V_{g}$ curves for $B$ increasing from 0 to $3.5 \mathrm{~T}$ in steps of $0.05 \mathrm{~T}$. We can see the evolution of the first 24 current peaks. The positions of the first three peaks depend monotonously on $B$, whereas the other peaks oscillate back and forth a number of times. The number of "wiggles" increases with $N$. Close inspection of the figure reveals that the current peaks generally shift in pairs with $B$. We see this even-odd effect up to $N=40$. Note, for instance, that around $3.5 \mathrm{~T}$ the peak spacing alternates between "large" for even $N$ and "small" for odd $N$. Intriguingly, just before entering the regime where they evolve smoothly with $B$, the peaks making up a pair move out of phase during the last one or two wiggles (see also Ashoori et al. in Ref. [2]).

For the simplest explanation of the magic number and the $B$ dependence we ignore, for the moment, the Coulomb interactions. The energy spectrum in a $B$ field

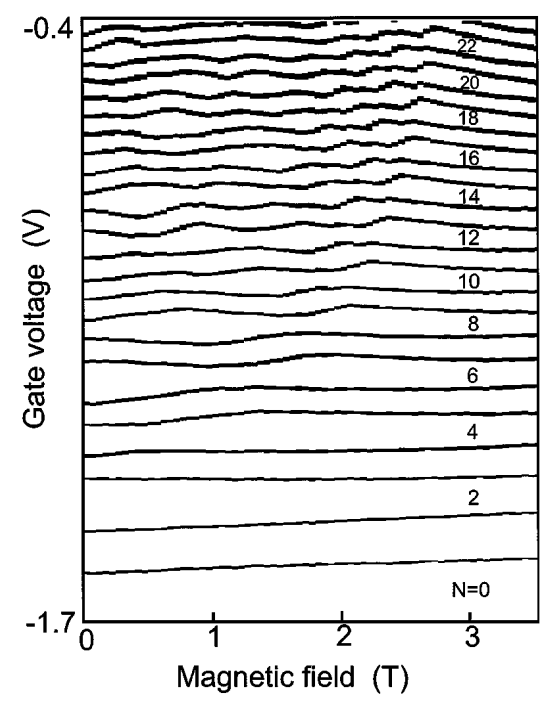

FIG. 2. Plot of the gate voltage positions of the current oscillations vs magnetic field for a dot with $D=0.5 \mu \mathrm{m}$. 
can be solved analytically for a dot with a 2D radial harmonic confining potential [7]. The energy $E_{n \ell}$ of a state with a radial quantum number $n(=0,1,2, \ldots)$ and angular momentum quantum number $\ell(=0, \pm 1, \pm 2, \ldots)$ is given by

$$
E_{n \ell}=(2 n+|\ell|+1) \hbar\left(\frac{1}{4} \omega_{c}^{2}+\omega_{0}^{2}\right)^{1 / 2}-\frac{1}{2} \ell \hbar \omega_{c},
$$

where $\hbar \omega_{0}$ is the electrostatic confinement energy and $\hbar \omega_{c}$ is the cyclotron energy. Spin is neglected so each state is twofold degenerate. At $B=0, E_{n \ell}$ has degenerate sets of states, which are separated by $\hbar \omega_{0}$ from each other and are completely filled for $N=2,6,12,20$, etc. These $N$ values can be regarded as magic numbers since they signify the complete filling of a shell. The unusually large addition energies we observe for $N=2$, 6 , and 12 are consistent with this picture. This shell structure should persist as long as the 2D lateral potential is radially parabolic to a fairly high degree and $\hbar \omega_{0}$ is comparable to, or larger than, the interaction energy. We believe that our vertical dot structures meet these conditions. However, as $N$ is increased, the potential can be significantly deformed by the effects of screening. This could be the reason why we observe the third harmonic shell only in some of the devices.

In Fig. 3(a) we plot $E_{n \ell}$ vs $B$ calculated for $\hbar \omega_{0}=$ $3 \mathrm{meV}$. A single-particle state with a positive or negative $\ell$ shifts to lower or higher energies, respectively, as $B$ is initially increased from $0 \mathrm{~T}$. The $B$-field dependence of these states gives rise to an addition energy for even $N$ that is strongly dependent on $B$. On the other hand, the addition energy for odd $N$ is determined only by the effect of Coulomb repulsion, which is responsible for lifting spin degeneracy. This should lead to the pairing of the conductance peaks, which is evident in Fig. 2. In Fig. 3(a) we mark the energy curve for the seventh and eighth electrons with a dashed line to illustrate that these electrons undergo transitions in their quantum numbers: $(n, \ell)$ goes from $(0,2)$ to $(0,-1)$ at $1.3 \mathrm{~T}$ and then to $(0,3)$ at $2 \mathrm{~T}$. These transitions are also seen in Fig. 2, demonstrating that $3 \mathrm{meV}$ is a reasonable value for the confinement potential. In a similar fashion, we can identify the quantum numbers of the other electron states. A more detailed comparison can be made from Fig. 3(b), which shows the $B$-field dependence of the fifth, sixth, and seventh peaks measured symmetrically from $B=-5$ to $5 \mathrm{~T}$. It is clear that the fifth and sixth peaks form a pair. At $1.3 \mathrm{~T}$ the sixth peak has a maximum, whereas the seventh peak has a minimum. This corresponds to the crossing of the third and fourth energy curves at $1.3 \mathrm{~T}$ in Fig. 3(a). For $\hbar \omega_{0}=3 \mathrm{meV}$, it follows that the effective dot diameter is about $100 \mathrm{~nm}$. At the $B$ field where the states last cross the single-particle states merge to form Landau levels. The single-particle excitation energy calculated, for example, at $B=3.5 \mathrm{~T}$, is, however, still large [between 1 and $1.5 \mathrm{meV}$, see Fig. 3(a)] and significantly contributes to the addition (a)

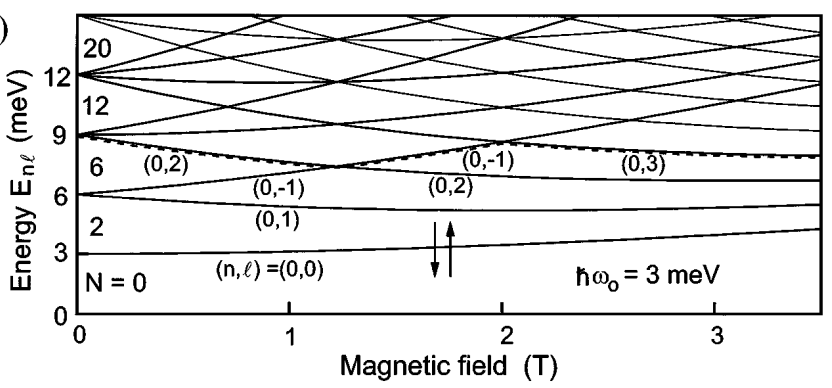

(b)

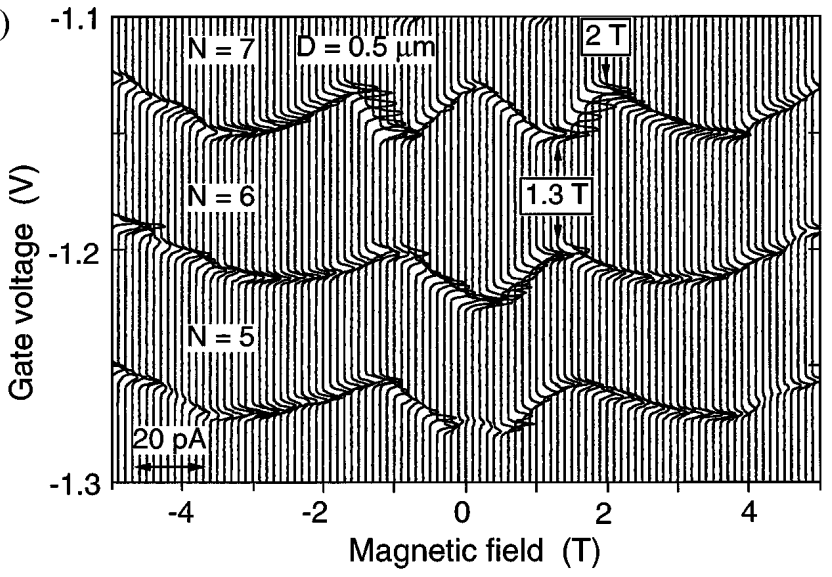

FIG. 3. (a) Calculated single-particle energy vs magnetic field for a parabolic potential with $\hbar \omega_{0}=3 \mathrm{meV}$. Each state is twofold spin degenerate. The dashed line is discussed in the text. (b) Evolution of the fifth, sixth, and seventh current peaks with $B$ field from -5 to $5 \mathrm{~T}$ observed for the $D=0.5 \mu \mathrm{m}$ dot. The original data consists of current vs gate voltage traces for different magnetic fields, which are offset and rotated by $90^{\circ}$.

energy for even $N$. This leads to the alternate peak spacings observed around 3.5 T in Fig. 2.

We now focus on spin filling in the second shell near $B=0 \mathrm{~T}$. Fig. 4(a) shows the $B$-field dependence of the third, fourth, fifth, and sixth current peaks. The pairing of the third and fourth peaks and the fifth and sixth peaks above $0.4 T$ is clearly seen. However, we intriguingly find that below $0.4 \mathrm{~T}$ the third and fifth peaks are paired, and the fourth and sixth peaks are paired. The evolution as a pair of the third and fifth peaks for $B<0.4 \mathrm{~T}$ is continued by the third and fourth peaks for $B>0.4 \mathrm{~T}$. Similarly, the evolution as a pair of the fourth and sixth peaks for $B<0.4 \mathrm{~T}$ is continued by the fifth and sixth peaks for $B>0.4 \mathrm{~T}$. This rearrangement of the pairing can be understood in terms of Hund's rule, which is well known in atom physics [3]. Hund's rule favors the filling of parallel spins up to the point where the shell is half filled, and we use this to derive the $B$-field dependence of the electrochemical potential $\mu(N)$ given in Fig. 4(b). In a constant interaction model we can simply add a constant energy $U$ to the energy of the single-particle states to derive $\mu(N)$. To include Hund's rule in our addition spectrum we introduce an energy $\Delta$, which represents the energy reduction due to exchange interactions between 
(a)

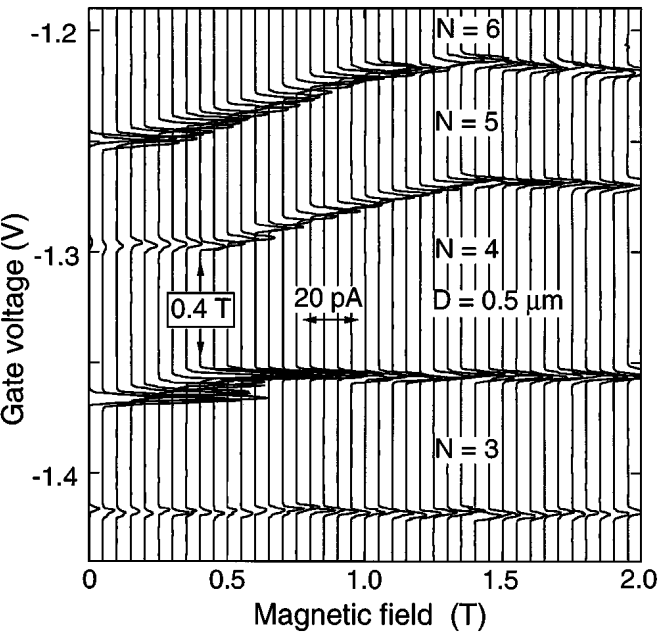

(b)

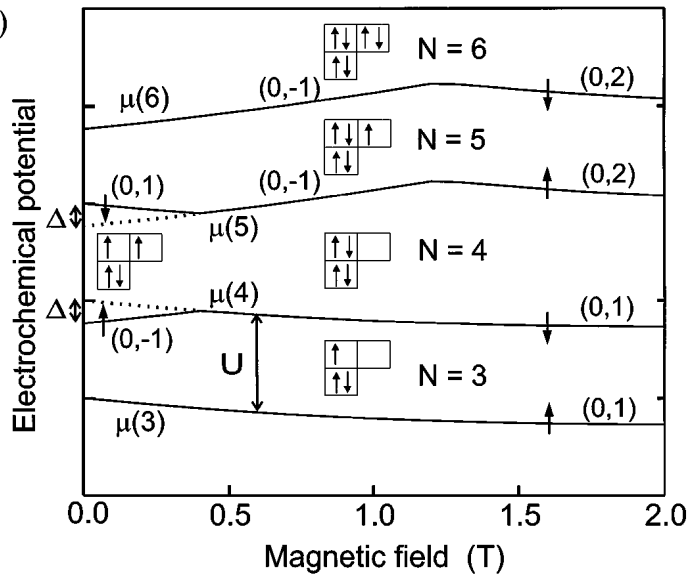

FIG. 4. (a) Evolution of the third, fourth, fifth, and sixth current peaks with $B$ field from 0 to $2 \mathrm{~T}$ observed for the $D=0.5 \mu \mathrm{m}$ dot shown in a manner described for Fig. 3(b). (b) Calculated electrochemical potential vs magnetic field for the model described in the text and parameters $U=3 \mathrm{meV}$, $\Delta=0.7 \mathrm{meV}$, and $\hbar \omega_{0}=3 \mathrm{meV}$.

electrons with parallel spins. Specifically for $N=4$, the ground state energy can be lowered if the outer two electrons have parallel spins with different angular momenta rather than antiparallel spins with the same angular momentum. $\mu(4)$ is reduced by an amount $\Delta$ and there is a corresponding increase in $\mu(5)$. This exchange effect is canceled in the presence of a $B$ field when the $(0, \pm 1)$ states, which are degenerate at $B=0 \mathrm{~T}$, are split by energy $\Delta$. This is the simplest way to account for exchange effects in a constant interaction model, however, for small $N$ we find a remarkable agreement between what we see in Fig. 4(a) and that predicted in Fig. 4(b) if we assume $U=3 \mathrm{meV}$ and $U=0.7 \mathrm{meV}$. In Fig. 4(b) we include quantum numbers $(n, \ell)$ to identify the angular momentum transitions and pictorial diagrams to illustrate the spin configurations. In our model, the addition energy for $N=4$ at $B=0 \mathrm{~T}$ is expected to be larger by $2 \Delta(=1.4 \mathrm{meV})$ than that for $N=3$ and 5 , and this is indeed observed in Fig. 1(b). Our model for including Hund's rule is very simple. More rigorous Hartree-Fock calculations, as performed in Ref. [8], are needed for a quantitative comparison. Very recently Tamura [8] and Eto [9] have actually been able to calculate addition spectra that closely reproduce our data when $\hbar \omega_{0}$ is comparable to or larger than the interaction energy.

For $B<0.4 \mathrm{~T}$, we also see an intriguing pairing in the height of the current peaks. The fourth and sixth peaks are higher than the third and fifth peaks. In addition, as the temperature is raised from $50 \mathrm{mK}$ to $1 \mathrm{~K}$ at $B=0 \mathrm{~T}$, the fourth and sixth peaks become small, while the third and fifth peaks gradually grow. We also note that similar behaviors have been observed for $N=9$ and 16 [see also the small maxima in Fig. 1(b)], which correspond to half filling of the third and fourth shells. These observations deserve further investigation.

In conclusion, we use single-electron tunneling spectroscopy to probe electronic states of a few electron vertical quantum dot atom. At zero magnetic field the addition energy reveals a shell structure associated with a 2D harmonic potential. As a function of magnetic field, current peaks evolve in pairs, due to the antiparallel filling of spin-degenerate states. Close to zero magnetic field, however, this pairing behavior is altered to favor the filling of states with parallel spins in line with Hund's rule.

We would like to thank H. Akera, L. Canali, X. Dauw, T. Oosterkamp, A. Koolen, P. van Son, S. Nair, Y. Tokura, and H. Tamura for their help and useful discussions. This work was initiated during a visit of S. T. to Delft in a collaboration program between Delft and NTT. We acknowledge G. Bauer for arranging this program. S. T. and L. P. K. thank the Nederlandse Organisatie voor Wetenschappelijk Onderzoek and the Royal Netherlands Academy of Arts and Sciences, respectively, for financial support.

[1] M. A. Kastner, Phys. Today 46, 24 (1993); R. C. Ashoori, Nature (London) 379, 413 (1996).

[2] R. C. Ashoori et al., Phys. Rev. Lett. 71, 613 (1993); T. Schmidt et al., Phys. Rev. B 51, 5570 (1995).

[3] L. I. Schiff, Quantum Mechanics (MacGraw-Hill, New York, 1949).

[4] S. Tarucha et al., Superlattices Microstruct. 18, 121 (1995); D. G. Austing et al., Semicond. Sci. Technol. 11, 388 (1996).

[5] M. Macucci et al., J. Appl. Phys. 77, 3267 (1995).

[6] J. Weis et al., Phys. Rev. Lett. 71, 4019 (1993).

[7] C. G. Darwin, Proc. Cambridge Philos. Soc. 27, 86 (1930); V. Fock Z. Phys. 47, 446 (1928).

[8] N. Johnson, J. Phys. Condensed Matter 7, 965 (1995); Y. Tanaka and H. Akera, Phys. Rev. B 53, 3901 (1996); H. Tamura (private communication).

[9] M. Eto (private communications). 